\title{
Research of Quantum Entanglement and Quantum Coherence Caused by Atomic Ensembles in Optical Cavity
}

\author{
Zhaohua Wang ${ }^{\text {a }}$ Liqing Ren, Hanying Wang \\ College of Energy Engineering, Yulin University, Shaanxi 719000, China \\ awangzhaohua841102@163.com
}

Keywords: atomic ensembles, quantum entanglement, quantum coherence

\begin{abstract}
As the most basic concept of quantum mechanics, a lot of researches on quantum entanglement and quantum coherence have been carried out in recent years. Based on this, the authors discuss in detail the quantum entanglement and quantum coherence generated by the atomic ensemble in circular cavity and optical mechanical cavities, and hope that the exposition content can bring inspirations for industry insiders.
\end{abstract}

\section{Introduction}

Based on the linear optical scheme, the practical applications of atomic ensembles belong to the focused field of academic focus in recent years [1]. The research that controls the laser field and the dissipation of the electromagnetic field to obtain the entanglement scheme belongs to the typical one [2]. In order to further promote the development of related fields, it is exactly the reason why this article conducts specific research on the quantum entanglement and quantum coherence generated by atomic ensembles in optical cavity [3].

\section{Imprisoned quantum entanglement and quantum coherence generated by atomic ensemble in a circular optical cavity}

\subsection{Atomic ensembles and Hamiltonian quantities}

It can be easily seen from the Fig. 1 that the system diagram shows the atomic ensembles placed in a high-quality annular cavity, where the two modes with opposite propagation directions and same frequency form the cavity field. Suppose $\vec{k} R=-\vec{k} L \equiv \vec{k} c$, we can get the annihilation operator $\hat{a} R$ in propagation mode of clockwise cavity field and the annihilation operator $\hat{a} L$ in propagation mode of anticlockwise cavity field. Table 1 supplements the information in Figure 1[4].



Fig.1 System diagram 
Table 1 Supplementary information

\begin{tabular}{ccccc}
\hline $\mathrm{R}$ & $\mathrm{L}$ & $\omega_{R}=\omega_{L} \equiv \omega_{c}$ & $\left|0_{j}\right\rangle,\left|1_{j}\right\rangle,\left|u_{j}\right\rangle,\left|s_{j}\right\rangle$ & $\vec{r} j$ \\
\hline $\begin{array}{c}\text { Clockwise } \\
\text { direction }\end{array}$ & $\begin{array}{c}\text { Anticlockwise } \\
\text { direction }\end{array}$ & $\begin{array}{c}\text { Coelenteron } \\
\text { frequency }\end{array}$ & The j-th atom & Position \\
\hline
\end{tabular}

The atomic ensembles consist of $\mathrm{N}$ identical four-level atoms. Thereinto, the four-level atoms interact respectively with the external driving field and the cavity field [5]. Figure 2 is the atomic level diagram and laser field and cavity field coupling structure chart. As can be clearly seen from the figure, this figure essentially shows the transitions of $R b$ originating from $F=1 \leftrightarrow F^{\prime}=1$. The total Hamiltonian quantities for the atoms and cavity modes are obtained by combining the calculations, that is $\hat{H}_{T}=\hat{H}_{0}+\hat{H}_{A L}+\hat{H}_{A C}$.In-depth calculations can obtain atomic ensembles with or without size effect. Difference is mainly reflected in the parameters and derived from Hamiltonian quantities.

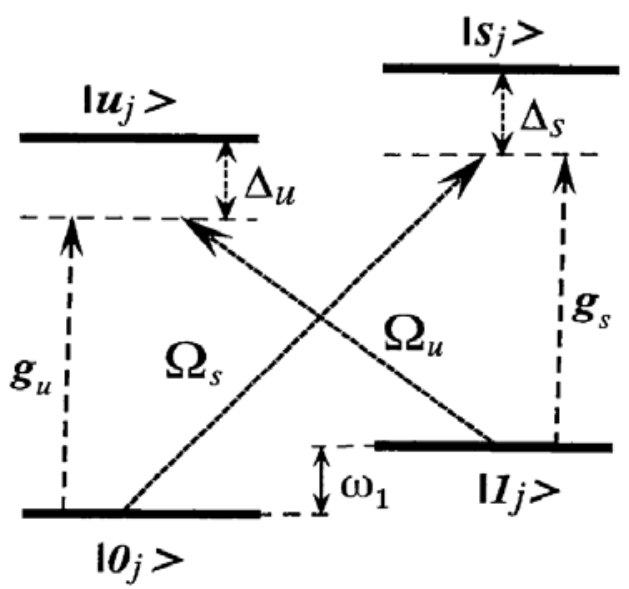

Fig. 2 Atomic energy level diagram and laser field and cavity field coupling structure chart

\subsection{Quantum entanglement and quantum coherence caused by finite size effect}

Coherence and correlation generated by finite-sized atomic ensembles also require attention, and the coherence and correlation are derived from cavity modes. Here we can use density operators to represent stable mixed-state systems, namely:

$$
\dot{\rho}=-\frac{i}{h}\left[\hat{H}_{e}, \rho\right]+\frac{1}{2} k \sum_{j=1}^{2}\left(2 \hat{a}_{j} \rho \hat{a}_{j}^{\mathrm{I}}-\rho \hat{a}_{j}^{\mathrm{I}} \rho \hat{a}_{j} \rho-\rho \hat{a}_{j}^{\mathrm{I}} \rho \hat{a}_{j}\right)
$$

The related information is shown in Table 2. The spontaneous emission of atoms can be effectively eliminated by selecting the large detuning between the laser and the cavity mode. The subsequent calculation can be used to obtain the average of the cavity field and the steady state of the correlation function Solution, namely:

$$
\left\langle\hat{d}_{j}^{2}\right\rangle=\frac{\lambda_{j}^{2}\left(k_{2}+\Omega_{j}^{2}\right)}{2 \omega_{0} h_{j}}
$$

Table 2 Related information

\begin{tabular}{ccc}
\hline$\hat{H}_{e}$ & $k$ & $\hat{a}_{j}^{\text {in }}(t)$ \\
\hline $\begin{array}{c}\text { Effective Hamiltonian } \\
\text { quantities }\end{array}$ & Cavity & Optical cavity inputs quantum noise \\
\hline
\end{tabular}




\section{Quantum entanglement and quantum coherence generated by atomic ensembles in optical mechanical cavity}

\subsection{Model and main equation}

Fig. 3 is system composed of optical lattice and laser driver. There are $\mathrm{N}$ nodes in the optical lattice. The waist length of cavity mode at the lattice is represented by ${ }^{\omega_{0}}$. Table 3 shows the necessary information for the system. The atoms placed on the nodes can also be regarded as a two-level system. The system consists of ground state $\left|g_{n}\right\rangle$ and excited state $\left|g_{n}\right\rangle$. Under normal circumstances the two-level system is real number. But $E_{L}$ will produce radiation pressure in the endoscope. At this time the mirror must be in a state of micro-vibration.

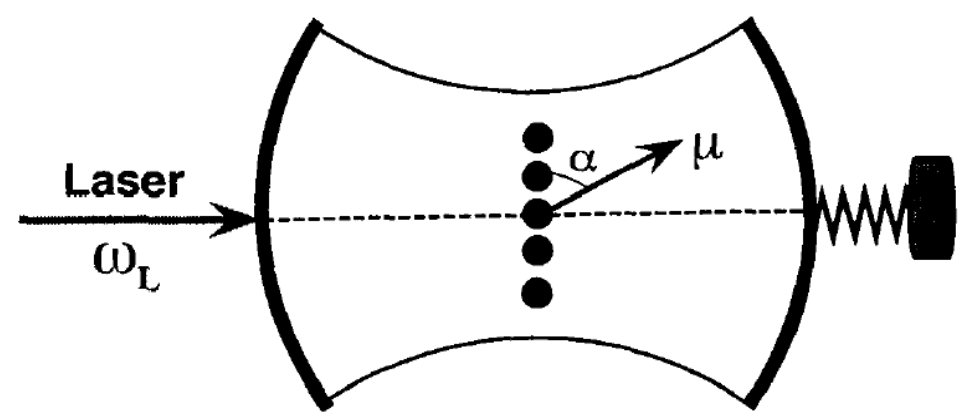

Fig.3 system composed of optical lattice and laser driver

Table 3 Necessary information

\begin{tabular}{cccccc}
\hline $\mathrm{d}$ & $\omega_{a}$ & $\vec{\mu}=\left\langle e_{n}\left|\vec{\mu}_{n}\right| g_{n}\right\rangle$ & $\mathrm{m}$ & $\omega_{m}$ & $E_{L}$ \\
\hline Nodal & Transition & Transition dipole & Mass & Resonant & Classical \\
distance & frequency & moment & & frequency & laser field \\
\hline
\end{tabular}

Carrying out in-depth calculations can obtain corresponding Hamiltonian quantities and cavity mode polarization direction of optical lattice excimer. Considering the optical lattice energy state, we can use the exciton modulo operator to obtain the free Hamiltonian quantities, the interactive Hamiltonian quantities. Finally, the relation between cavity annihilation operator and polaron annihilation operator is obtained, that is $a=\Phi \cos \phi-\Psi \sin \phi$. From this it can be concluded that $\omega_{c}=\omega_{1}=\omega_{0}$, which represents the resonance of the cavity frequency with the exciton frequency.

\subsection{Linear fluctuation analysis}

Combining the polaron and oscillating mirror operator Heisenberg motion equation, the damping term is introduced to obtain the laser frequency under the rotating frame. Assuming that the laser field resonates with the cavity field and the atom, the non-zero second-order moment can be obtained:

$$
\frac{1}{2}\left(\left\langle\xi(t) \xi\left(t^{\prime}\right)\right\rangle+\left\langle\xi\left(t^{\prime}\right) \xi(t)\right\rangle\right)=\left(\bar{n}+\frac{1}{2}\right) \delta\left(t-t^{\prime}\right)
$$

The steady-state solution of the harmonic oscillator variables can be obtained by further calculation and $G_{\Psi}=\sqrt{2}\left(\frac{1}{2} G \Psi_{s}-G_{s} \Psi_{s}\right)$ is obtained. Table 4 shows the relevant information in the formula.

The effective coupling constant of the polaron to the cavity field. 
Table 4 Related information in the formula

\begin{tabular}{llll}
\hline$\xi(t)$ & $k_{B}$ & $G_{\Psi}$ & $G_{\Phi}$ \\
\hline $\begin{array}{l}\text { Quantum } \\
\text { noise }\end{array}$ & Brown & $\begin{array}{l}\text { The effective } \\
\text { coupling constant } \\
\text { of the polaron } \Psi \text { to } \\
\text { the cavity field }\end{array}$ & $\begin{array}{l}\text { The } \\
\text { coupling constant } \\
\text { of the polaron } \Phi_{\text {to }} \\
\text { the cavity field }\end{array}$ \\
\hline
\end{tabular}

\subsection{Quantum entanglement produced by atomic ensemble in optical mechanical cavity}

Polaron-oscillator coupling, entanglement and compression, inequality Cauchy-Schwartz and self-correlation function, compression base all belong to the content about quantum entanglement generated by atomic ensembles in optical mechanical cavity. For example, the polarizer-oscillator mirror coupling needs to analyze the coupling between the polaron and the oscillatory mirror. And after calculation, it can be found that the same frequency coupling between two will result in monochromatic entanglement; The entanglement and compression need to analyze the entanglement and compression between polarons $\Psi$ and oscillator b. The specific analysis process needs to introduce the anti-phase and in-phase quadrature components of the fluctuation operator to determine the necessary conditions for the entanglement. Inequality Cauchy-Schwartz and self-correlation analysis mainly focus on a concise method. The combination of the two can determine the entanglement state between polaron mode $\Psi$ and oscillation mirror b more quickly. The compression base needs to carry out analysis by combining noise two-photon correlation function and the state of the compressed base. Finally, the conclusion shown in Table 5 can be drawn.

Table 5 Related conclusion

\begin{tabular}{ll}
\hline Conclusion & Information \\
\hline Polaron $\Psi$ and shock mirror have the & Produce monochrome entanglement \\
same frequency & Produce entanglement \\
$\bar{n}<1$ & Produce entanglement \\
$\bar{n}<1 、 G_{\Psi}<2 \gamma$ & \\
\hline
\end{tabular}

\subsection{Three-mode quantum entanglement and quantum coherence}

Analysis around two-color entanglement and coupled non-degenerate modes needs to be conducted; The two-color entanglement can draw a conclusion that whether or not the Cauchy-Schwartz inequality violates, it is unavoidable to occur entanglement in the range of U; Coupling non-degenerate modes can analyze the influence of the interactions types on the entanglement of the polarization modes entanglement, that is, $U$ and ${ }^{G_{t}}$ are comparable with the time when the entanglement occurs, and the size of $U$ and $G_{t}$ is directly affected by $U$.The increase of $U$ will enhance entanglement and the decrease of $U$ will weaken entanglement.

\section{Conclusions}

In summary, the research on quantum entanglement and quantum coherence generated by atomic ensemble in optical cavity possesses high practical significance. On this basis, the quantum entanglement and quantum coherence and linear fluctuation analysis caused by the finite size effect discussed in this paper prove the practical value of the research. Therefore, the content of this article can play a certain reference role in the related field of theoretical research and practical exploration. 


\section{Acknowledgements}

This work was supported by Shaanxi science and technology research and development plan project:2016GY-196; Shaanxi science and technology research and development plan project: 2014K05-11.

\section{References}

[1] Ali Ü. C. Hardal, Özgür E. Müstecaploglu. Spin squeezing and quantum coherence in systems with two-particle exchange interactions[J]. 2013.

[2] Verhagen E, Deleglise S, Weis S, et al. Quantum-coherent coupling of a mechanical oscillator to an optical cavity mode[J]. Nature, 2012, 482(7383):63-7.

[3] Li T, Yang G J, Deng F G. Entanglement distillation for quantum communication network with atomic-ensemble memories[J]. Optics Express, 2014, 22(20):23897.

[4] Shi Z, Chen X, Xiang S, et al. Deterministic Generation of Macroscopic Entangled Coherent States for Distant Atomic Ensembles in Coupled Cavities[J]. International Journal of Theoretical Physics, 2014, 53(11):3728-3736.

[5] Su Q P, Liu T, Yang C P. Transferring multipartite entanglement among different cavities[J]. Quantum Information Processing, 2016, 15(1):1-17. 